\title{
Reinhard Marx Anforderungen an ein europäisches Asylrecht
}

\section{Europa am Scheideweg}

Mit der Einheitlichen Europäischen Akte von 1986 wurde Art. $8 \mathrm{a}$ in den Vertrag der Europäischen Gemeinschaften (EG) eingeführt, demzufolge innerhalb des Gemeinschaftsgebietes bis zum 31. Dezember 1992 schrittweise Kontrollen von Gütern. Personen, Dienstleistungen und Kapital abgeschafft werden sollen. Bereits in ihrem Weißbuch vom Juni 198 s hatte die Kommission vorgeschlagen, bis 1992 Grenzkontrollen gegenüber EG-Bürgern abzubauen'. In ihrer Kommunikation vom Mai 1992 machte die Kommission darüber hinaus deutlich, daß nach ihrer Ansicht sämtliche Binnenkontrollen abgeschafft werden sollten. Denn es solle ein wirklicher interner Markt errichtet werden, der wie ein nationaler Markt funktioniere. Dics habc zur Folge, daß sämtliche Kontrollen an internen Grenzen abzuschaffen seien, genauso wie es im nationalen Markt zwischen einzelnen Regionen keine Grenzkontrollen $\mathrm{gcbc}^{2}$.

Diese Entwicklung hat Auswirkungen auf die Flüchtlinge und Asylsuchenden, die nach Europa kommen wollen oder bereits hier leben. Zwei brcite Politikfelder haben sich seit Mitte der achtziger Jahre entwickelt, die unmittelbar mit der Asylfrage zusammenhängen: Einerseits sollen durch gemeinsame Maßnahmen unkontrollierte Wanderungen von Asylsuchenden und Flüchrlingen inncrhalb des Gemeinschaftsgebietes sowie gleichzeitige oder hintercinander geschaltete Mehrfachanträge von Asylsuchenden in verschiedenen EG-Staaten verhindert und andcrerseits sollen die Außengrenzen der EG nach einheitlichen und für allc Staaten geltenden Regeln gesichert werden. Dic erste Aufgabc erfordert die Festlegung eines einzigen Staates zur Behandlung und Entscheidung über Asylanträge. Zur Regelung dieser Frage licgen bereits unterzeichncte, aber noch nicht in Kraft getrctene Abkommen vor. Dic Kontrolle der Außengrenze verlangt demgegenüber eine gemeinsame Visapolitik und einheitliche Kriterien zur Einreisekontrolle. Auch insoweit sind Abkommen entwickelt worden, die ebenfalls noch nicht in Kraft getreten oder noch nicht unterzeichnet sind. Der Vertrag über dic politische Union von Maastricht sicht zudem in Art. rcoc einc gemcinschaftsrechtliche Regelung der Visapolitik vor.

Die europäischen Staaten sehen sich mit einer Rechtsschutzkrise konfrontiert. Sie ist insbesondere das Ergebnis jahrelanger nationalstaatlich isolicrter Asylpolitiken in einer Situation, in der gemeinsames Handeln gefordert gewesen wäre. In nahezu allen europäischen Staaten steigen die Flüchtlingszahlen, werden die Aktenberge

\footnotetext{
1 Commission of the European Communities, Completing the Internal Market. White Paper from the Commission to the European Council, Jun 1985, Rdn. 55 .

2 Commission des Communautes Europeennes, Commumcation v. 5. 5.1992-0/92/120, Anhang 1, Nr. 3, S.9.
} 
infolge unbearbeiteter Asylanträge zusehends größer und schlagen gesetzliche und administrative Lösungsversuche regelmäßig fehl. Zwar ist Europa nur am Rande vom Weltflüchtlingsproblem betroffen ${ }^{3}$, doch darf die Wirkung dieser Fehlschläge auf Öffentlichkeit und Gesellschaft nicht unterschätzt werden. Diskriminierungen, Stigmatisierungen und kriminelle Übergriffe meist jugendlicher Täter gegen Asylsuchende sind die bedrohlichen Folgen dieser politischen Versäumnisse.

Was sind die Ursachen der europäischen Asylkrise? Da sind zunächst die Zahlen. Besorgniserregend sind nicht so sehr die tatsächlichen Zahlen als vielmehr deren öffentliche Wahrnehmung sowie dic hieran anknüpfende politische Instrumentalisierung.

Zwar ist ein signifikanter Anstieg der Asylbewerberzahlen in Westeuropa festzustellen. Der Aulas-Bericht des Europäischen Parlaments nennt andererseits I7,5 Millionen Flüchtlinge weltweit. Während in den siebziger Jahren die weltweite Flüchtlingszahl von 2 auf 8,5 Millionen Personen angestiegen ist, hat sie sich Anfang der neunziger Jahre damit noch einmal verdoppelt. Darüber hinaus wird die Zahl der internen Flüchtlinge auf 24 Millionen geschätzt. Allein in Äthiopien, Mosambique und im Sudan befinden sich innerhalb der Staatsgrenzen sechs bis sieben Millionen Menschen auf der Flucht's. Verschärfend kommt hinzu, daß es für diese Flüchtlinge keinen entwickelten Rechrsschutz gibt. Demgegenüber fallen die o,s Millionen Flüchtlinge des Jahres $199 \mathrm{r}$ in Westeuropa zahlenmäßig kaum ins Gewicht.

Ein weiteres Phänomen des europäischen Flüchtlingsproblems ist das seit 1982 und 1983 zu beobachtende Phänomen der sog. irregulären Einreise von Flüchtlingen und Asylsuchenden. Über eine Million Flüchtlinge haben seit 1983 auf diese Weise um Schutz nachgesucht ${ }^{6}$. Jede europäische Regierung neigte jedoch dazu, das Problem aus ihrer eigenen beschränkten Perspektive zu betrachten sowie auf Kosten des Nachbarn Lösungen zu suchen. Es entwickelte sich ein Trend, Asylsuchenden auf Kosten der Nachbarstaaten durch eine enge Auslegung des Prinzips des ersten Asyls den Zugang zum Verfahren abzuschneiden. Asylpolitik bestcht in Europa daher vorrangig in der Umwälzung der Last entweder auf einen Nachbarstaat oder auf das Ersttransitland'. Diese Politik der Versagung des Zugangs zum Verfahren entwikkelre sich jedoch bereits in den siebziger Jahren, als sich in Europa das Phänomen der umberreisenden Flüchtlinge (refugees in orbit) entwickelte.

Zwei weitere Phänomene hängen eng mit der irregulären Einreise der Asylsuchenden nach Europa zusammen, nämlich die ungelöste Migrationspolitik sowie der extraregionale Charakter des europäischen Flüchtlingsproblems. Zum ersten Aspekt wird darauf hingewiesen, daß zu den 600000 legalen Einwanderern, die jedes Jahr nach Europa kommen, die Zahl der Asylsuchenden hinzugezählt werden müsse9. Während 1978 6,5\% aller Zuwanderer Asylsuchende und Flüchtlinge gewesen seien, hätte deren Anteil an der Gesamtquote der Zuwanderung nach Europa 1988 34\% ausgemacht ${ }^{10}$. Die Zahl derjenigen, die über das Asylverfahren eine sonst nicht erreichbare Migration anstreben, ist gewiß signifikant. Dies rechtfertigt aber nicht die

3 Gil Loescher, The Europenn Community and refugees, in: International Affairs $1989,618$.

4 DOC EN/PR/i is635, 20.9.1991, S.7.

S Analytical Report of the UN-General Secretary on internally displaced persons, E/CN.4/1992/23, 14. 2. 1992 , Nrn. s u. 37 f.

6 Gil Loescher, a. a. O., S. $618 \mathrm{f}$.

7 Alexander Casella, Das Asylproblem - eine Herausforderung für Europa, Europa-Archiv 1988, S. 377 .

8 Goran Melander, Refugec's in Orbit, in: ai ( $\mathrm{Hg}$ ), Bewahrungsprobe fur ein Grundrechr, Baden-Baden 1978, S. 71 .

9 Alcxander Casella, a. a. O., S. 379

to Alexander Casella, a. a. O. 
Politik der Innenverwaltungen, das Asylrecht vorrangig als Instrument zur Abwehr unerwünschter Einwanderungen zu behandeln.

Die rapide Zunahme der irregulär einreisenden Asylsuchenden scit 1 982 hat zu einer erheblichen Belastung der nationalen Asylverfahren in Europa und zu zunehmenden Defiziten im System des Rechtsschutzes geführt ". In seinem Bcricht des Jahres 1987 äußerte der UNHCR daher seine Besorgnis über die zunehmende Anwendung restriktiver Kriterien und Maßnahmen in Europa, die "schrittweisc das Prinzip des Asyls aushöhlen und langfristig die internationale Koordination und Kooperation in diesem Bereich paralysiercn « würden ${ }^{12}$. Auch in seinem Bericht für 1992 kritisierte der UNHCR, daß Abwchrmaßnahmen gegen illegale Einwanderung das Risiko der Zurückweisungen von Flüchtlingen erhöhten'3. Der kooperative Geist der siebziger Jahre in Europa ist durch das "Gesetz des Dschungels " ersetzt worden, und cs ist eine chaotische und sich zunehmend verschlechternde Situation festzustellen, auf die der Begriff der »europäischen Flüchtlingskrise zutrifft ${ }^{14}$. Die Abschottungspolitiken aller europäischen Regierungen haben dazu geführt, daß man von der "Festung Europa" sprechen kann's.

Bis Ende der achtziger Jahre wurde der extraregionale Charakter des europäischen Flüchtlingsproblems, d.h. die übcrwiegende Zahl von außereuropäischen Asylsuchenden betont. Gegen deren irreguläre Wanderungen wurden vorrangig Abschottungspolitiken entwickelt. Demgegenüber waren bis zu diesem Zeitpunkt Asylsuchende aus Osteuropa politisch willkommen. Heute herrscht angesichts der Umkehrung des Trends dagegen Furcht vor einem dramatischen Anstieg der Flüchtlingszahlen aus Osteuropa. Hiergegen wird eingewandt, daß die derzeitige Welle von Ausländerfeindlichkeit im Westen einer Zunahme der Ost-West-Migration entgegenstehe $^{16}$. Auch gebe es eine Migrations- und Flüchtlingsbewegung innerhalb der ostcuropäischen Region und sei die isolierte Fixierung allein auf die Ost-WestMigration nicht gerechtfertigt ${ }^{17}$.

Auf Veranlassung der westlichen Staatengruppe befaßte sich bereits Mitte der achtziger Jahre das Exekutivkomitee des Programms des UNHCR mit der Fragc der irregulären Einreise von Asylsuchenden und gab eine Studie zu dieser Frage in Auftrag. Der vom UNHCR Beauftragte warnte in seiner Studic u. a. davor, das Problcm der irregulären Einreise durch isolierte technisch-administrative Maßnahmen lösen zu wollen. Vielmehr müßten langfristig die Fluchtursachen bekämpft werden ${ }^{18}$. Bcreits in seiner von der UN-Menschenrechtskommission in Auftrag gegebenen Studie über Massenfluchtbewegungen hatte der frühcre UNHCR, Prinz Sadruddin Aga Khan, auf die Kombination von massiven Menschenrechtsverletzungen, Bürgerkriegen und ökonomischen Mißständen als Fluchtursachen hingewie$\operatorname{sen}^{\prime 2}$, und auch der UN-Generalsekretär weist in seinem erwähnten Bericht auf diese

is Gil Loescher, a. a. O., S.6rg.

12 Ansprache des United Nations High Commissioner for Refugees (UNHCR) v. s. 10.1987 an den 3. Hauptausschuß der UN-Generalversammlung; UNHCR, A/AC.96/INF. 174, 4.7.1985, Nr. 20.

${ }_{13}$ UNHCR, E/1992/65, 20.5. 1992, Nr. 148 .

14 Goran Melander, Basic Differences in Refugee Policy in Western Europe and North America, In Defense of the Alien, Bd. 9, Lydia F. Tnması, New York 1987, S. 10, 102.

is Peter Nobel, Protection of Refugecs in Europe as seen in 1987, Raoul Wallenberg Institut, Bericht Nr. 4, Lund 1987, S. 34

I6 Marek Okolski, Migratory Movements from countries of Central and Eastern Europe, Council of Europe, MMP (91) rev., S. 24 l.

17 John Salt, Current and Future International Migration Trends affecting Europe, Council of Europe, $\mathrm{MMG}_{-4}(9 \mathrm{i})$ i, S. 17.

18 Gilbert Jaeger, Study of Irregular Movements of Asylum Seekers and Refugees, WG/M/3, X.8.1985, Rdn. 289-291.

19 UN, ECOSOC, Srudy on Hunan Rights and Massive Exoduses, Sadruddin Aga Khan, E/CN.4/igo3, 31. 12. 1981, Rdn. 2, 36 u. 11s. 
Verbindung der Fluchtursachen hin (Rdn. 38). Die UN-Generalversammlung hat seit 198 I deshalb in mehreren Erklärungen ihrer Besorgnis über Massenfluchtbewegungen Ausdruck verliehen ${ }^{20}$. Die EG-Regierungen haben jedoch einzelne Aspekte aus dem Zusammenhang herausgelöst und technischen Lösungen zuzuführen versucht.

\section{Der kurze Atem der Politik}

Bei Harmonisierung im EG-Bereich denkt der unbefangene Beobachter unmitrelbar an die Politik der EG-Institutionen. Zunächst sollte das gemeinsame Asylrecht auch gemeinschaftsrechtlich entwickelt werden. Es hätte dann das Europäischc Parlament mitbeteiligt wcrden müssen, so daß in einem gewissen Umfang Öffentlichkeit hätte hergestellt werden können. Eine derartige politische Lösung hatte die Kommission bereits in ihrem Weißbuch von 1985 angekündigt. Denn sie wollte einen Richtlinienentwurf zur Koordination der Regelungen zum Asylrecht erarbeiten. 1987 legte das zuständige Direktorat der Kommission den französischen Text cines derartigen Richtlinienentwurfes vor, der zwar nicht sonderlich von den noch zu erörternden Vorstellungen der Innenverwaltungen abwich, jedoch eine Gemeinschaftsstruktur zur Beratung der nationalen Regierungen und zur Entwicklung gemeinsamer Standards enthielt. Auf dem EG-Gipfeltreffen in Rhodos im Dezember 1988 wurde jedoch die Kompetenz der EG in Asylfragen bestritten. Die Regierungen richteten statt dessen die Gruppe der Koordinatoren ein, welche den ohnehin schon bestehenden zwischenstaatlichen Diskussions- und Abstimmungsprozeß lenken und unterschriftsreifen Abkommen zuführen sollte.

Die aus hohen Beamten der EG-Regierungen bestehende Gruppe der Koordinatoren legte im Juni 1989 dem Rat in Madrid einen Bericht vor, der ein Maßnahmenbündel zur Regelung der Asylfrage enthielt (sog. Palma-Dokument). Die eigentliche Arbeit war allerdings in der sog. Ad-hoc-Gruppe der Einwanderungsminister, welche im Oktober 1986 eingerichtet worden war, geleistet worden. Diese Gruppe ist aus der sog. TREVI-Gruppe hervorgegangen, welche 1975 in Rom eingerichtet worden war und sich mit Fragen der Kriminalitäts- und Terrorismusbekämpfung befaßt. Aus dem Palma-Dokument sind inzwischen zwei Abkommen hervorgegangen. Das erste Abkommen legt den allein zuständigen Asylstaat fest und wurdc am is. Juni r 990 in Dublin unterzeichnet. Es wird fälschlicherweise häufig "Dubliner Asylkonvention " genannt. Doch diese Konvention legt lediglich nach objektiven Kriterien den Staat fest, der innerhalb der EG allein für die Behandlung cines Asylbegehrens zuständig ist, enthält aber keinen Anspruch auf Asyl. Sie wird beherrscht vom Prinzip der einen Chance sowie vom Verursacherprinzip. Der Staat, der ein Visum ausgestellt hat oder über dessen Grenze der Asylsuchende illegal eingereist ist, hat die alleinige Zuständigkeit (Art. 3 ff.) für die Behandlung des Asylantrags. Er ist verpflichtet, den Asylsuchenden zu übernehmen, wenn dieser in einen anderen Staat zur Asylsuche weitergereist ist. Jedoch haben die Bundesrepublik, Frankreich und die Niederlande in den Beratungen auf einen nationalen Vorbchalt gedrängt, der es ihnen ermöglicht, ungeachtet der Zuständigkeit cines anderen Vertragsstaates das Asylgesuch zu behandeln (Art. 3 Abs. 5).

20 Res. $35 / 124$ v. 11. 12.1980, Res. $36 / 148$ v. 16.12.1981, in: VN 1982, 72: Res. $37 / 121$ v. 16.12.1982; Res. $38 / 1983$ v. 15. 12. 1983, in: VN 1984, 177 ; Res. $39 / 100$ v. 14. 12. 1984 , in: VN 1985, 134 ; Res. $40 / 166$ v. 16. 12. 1985, in: VN 1986, 116 ; Res. $41 / 70$ v. 3.12.1986, in: VN $1987,33$. 
Bislang haben lediglich Dänemark, Griechenland und das Vereinigte Königreich die

Konvention ratifiziert. Sie kann crst nach der Ratifizierung durch alle EG-Staaten in Kraft treten (Art. 22 Abs. 3). Überdies liegt der Brüsseler Text des Entwurfes eines Übereinkommens der EG-Staaten über das Überschreiten der Außengrenzen vom 24. Juli 1991 (Grenzübertrittskonvention) vor. Er regelt u. a. eine gemeinsame Visapolitik (Art. $17 \mathrm{ff}$.) und die Verpflichtung, Sanktionen gegen Beförderungsunternehmen einzuführen (Art. ${ }_{4}$ Abs. 2), enthält also generell Maßnahmen zur Kontrolle der EG-Außengrenzen. Die Unterzeichnung scheitert bislang am Streit zwischen Spanien und dem Vereinigten Königreich über die Zuständigkeit für die Außengrenzen in Gibraltar. Beide Abkommen sehen im übrigen vor, daß ein Verwaltungsausscluuß $\beta^{21}$ ihre Anwendung koordiniert und Streitfälle entscheidet. Einc intcrnationale gerichtliclie Kontrollc ist ausgeschlossen.

Parallel zur Etablierung einer gemeinsamen Asylpolitik der EG-Staaten hat sich seit $19 \$_{4}$ ein harter Kern besonders eifriger Europaverwalter herausgcbildet, nämlich die Schengen-Gruppe. Bereits am 1 3. Juli 1984 hatten Frankreich und Deutschland in Saarbrücken don schrittweisen Abbau von Grenzkontrollen zwischen ihren Staaten vereinbart. Am 14.Juni 198 s schlossen sich die Benelux-Staaten in dem kleinen luxemburgischen Ort Schengen diesem Unternehmen in einem gemeinsamen Vertrag an. Inzwischen hat die aus den Einwanderungsministern der fünf Staaten gebildetc Arbeitsgruppe ein Zusatzabkommen über den schrittwcisen Abbau der Grenzkontrollen (Schengener Zusatzabkommen) vorgelegt, das am 19. Juni 1990 unterzeichnet worden ist. Auch dieses enthält einen nationalen Vorbehalt (Art. 29 Abs.4) und überträgt die Überwachung einem Verwaltungsausschuß (Art. 13 I ff.). Schon die Personenidentität zwischen der Schengener und der EG-Arbeitsgruppe, aber erst recht der nahezu wortgleiche Text des Schengener Zusatzabkommens mit der Dubliner Konvention und dem Brüsseler Entwurf der Grenzübertrittskonvention machen deutlich, daß Schengen Pilotfunktion für den europäischen Entwicklungsprozcß hat.

Inzwischen sind Italien am 27. November r990 sowie Spanien und Portugal am 25. Juni 1991 dem Zusatzabkommen beigetreten. Mit Dänemark, Griechenland und dem Vereinigten Königreich finden derzeit Verhandlungen über einen Anschluß statt. Lediglich Irland fehlt also noch. Bemerkenswert ist im übrigen, daß die Bundesregierung bislang lediglich mit Blick auf Schengen das Ratifizierungsverfahren eingeleitet hat ${ }^{22}$ und dicser Mangel in der Schengendebatte des Bundestages ${ }^{23}$ von keinem der Redner problematisiert wurde. Lediglich Frankreich, Portugal und Spanien haben bislang das Schengener Zusatzabkommen ratifiziert ${ }^{24}$. Andererseits legt der Vertrag über die politische Union in Art. $100 \mathrm{c}$ des EG-Vertrages eine gemeinschaftsrechtliche Zuständigkeit für die Visapolitik fest und wird im Titel VI, Art. K r ff. das Asylrecht zur "Frage des gemeinschaftlichen Interesses erklärt. Allerdings sollen insoweit keine gemeinschaftsrechtlichen Lösungen, sondern zwischenstaatliche Konventionen entwickelt werden, welche allerdings die Zuständigkeit des Europäischen Gerichtshofes begründen können (Art. K I (7) bis (9) Abs. c). Unklar bleibt, ob und inwieweit nach Verabschiedung von Konventionen im Rahmen von Art. K I die bereits entwickelten Abkommen weiterhin Geltung haben werden. Hinzu kommt, daß im Rahmen des Europarates der Entwurf eines Abkom-

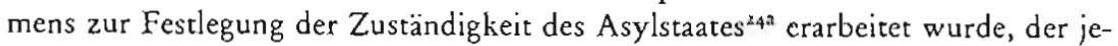

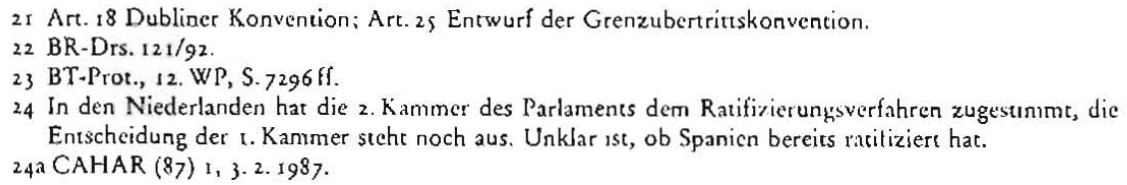


doch mit Rücksicht auf die politische Schubkraft der EG nicht weiter entwickelt worden ist. Die Situation ist also verworren, aber noch offen für angemessene politische Lösungen.

\section{Unabdingbare Schlüsselelemente einer europäischen Asylpolitik \\ 3.I. Zum Begriff der "Harmonisierung *}

Eine Harmonisierung der europäischen Asylpolitik setzt eine tatsächliche Vereinheitlichung der nationalen Asylgcsetzgebungen voraus, und zwar nach Maßgabe konsensfähiger Kriterien. Die Kommission hat demgegenüber bekräftigt, daß eine Vereinheitlichung materieller und formaler Standards erst langfristig in Angriff genommen werden könnte. Hingegen sollten bcrcits jetzt die Verfahren beschleunigt und ein gemeinsamer Begriff der "offensichtlich unbegründeten Asylbegehren" sowie des »sicheren Erstaufnahmelandes « und des "sicheren Herkunftsstaates* entwickelt werden ${ }^{25}$. Die Einwanderungsminister unterstützen diese Zielsetzungen in ihrem in Maastricht angenommenen Bcricht im wesentlichen, betonen aber zugleich eine Priorität der Harmonisierung der materiellen Kritcrien nach Maßgabc von Art. I A GFK. Hingegen erscheine eine prozessuale Harmonisierung weniger dringlich $^{26}$. Würdc zu starkes Gewicht auf eine derartige Harmonisierung gelegt, könnte der gesamte Harmonisierungsprozeß zum Erliegen kommen, denn dic Materic sei komplex und die Rolle von Gerichten berühre fundamentale Fragen der Staatsorganisation. Das Gebot der prozessualen Harmonisierung crfordere deshalb lediglich Abkommen zur Länge der Verfahren und zur prozessualen Behandlung "offensichtlich unbcgründeter Asylanträgc «. Priorität habe aber die materielle Harmonisierung (S. 32).

Harmonisierung des Asylrcchts heißt demnach lediglich Entwicklung gemeinsamer administrativ-technischer Kriterien zur Behandlung "offensichtlich unbegründeter Anträge sowie zur Regelung der Fragen des "sicheren Erstaufnahmelandes" und nsicheren Herkunftsstaatcs«. Während die Kommission in ihrer Kommunikation wenigstens noch die Notwendigkeit von individuellen Schutzgarantien bei der Gestaltung von Sondervcrfahren unterstreicht (S. s), berufen die Einwanderungsminister sich in ihrem Bericht zwar auf internationale Standards, jedoch ohne deren Verfahrensgarantien auch nur mit einem Wort zu errwähnen (S. 38-40). Es berührt seltsam, daß bei der Gestaltung eines gemeinsamen, die nationalen Interessen überhöhenden Prozesses gcrade dann, wenn es um die Gestaltung von Rechtsschutzgarantien geht, die nationalen Besonderheiten, sprich die nationale Staatssouveränität, bemüht werden. Es ist eine Binsenweisheit, daß ein Recht ohne Durchsetzungsmechanismen ohne Wert ist. Daher kommt der Harmonisierung gerade der Verfahrensgarantien bei der Entwicklung einer gemeinsamen Asylpolitik überragende Bedeutung zu. Politische Durchschlagskraft kann eine derartige Harmonisierung aber nur erlangen, wenn konsensfähige, also internationale Standards ausfindig gemacht werden können.

2s Commission of the European Communities, Communication from the Commission to the Council and the European Parliament on the right to asylum, SEC (91) 1857 final, 11. 10. 1991, S. 5-7.

26 Group Ad Hoc Immigration, Report from the Ministers responsible for immigration to the European Council meeting in Maastricht on immigration and asylum policy, SN 4038/91/WGI 930), 3.12.1991, S. 4 U. 14 . 


\subsection{Das völkerrechtliche Zurückweisungsverbot}

Nach allgemeiner Ansicht garantiert das Völkerrecht keinen Rechtsanspruch auf Asyl. Allenfalls wird insoweit von einer moralischen Verpflichtung der Staaten gesprochen ${ }^{27}$. Auch die Entwicklung des Vertragsvölkerrechtes seit 192 I hat nicht zu einer Anerkennung eines derartigen Anspruchs geführt. Jedoch enthält Art. 33 I der Genfer Flüchtlingskonvention eine fundamentale verfahrensrechtliche Schutznorm für Flüchtlinge. Während früher die Ansicht vertreten wurde, diese Norm schütz.e nur die legal oder illegal eingercisten Flüchtlinge vor Abschiebungen in ihr Herkunftsland, enthalte jedoch kein Zurückweisungsverbot für jene an der Grenze ${ }^{28}$, ist heutc allgemein anerkannt, daß Art. 33 I GFK auch Asylsuchende an der Grenze vor Zurückwcisung in ihr Herkunftsland schützt ${ }^{29}$. Inzwischen hat dieses Verbot gewohnheitsrechtlichen Rang erhalten ${ }^{30}$. Der UNHCR spricht ihm sogar den Charakter von jus cogens zu, so daß auch durch spätere entgegenstehende Übung der Inhalt und die Schutzwirkung des Refoulement-Verbotes nicht mehr verändert werden könnte.

Zwar wird behauptet, die GFK enthalte keine verbindlichen Leitlinien darüber, welche verfahrensrechtlichen Pflichten die Staaten gegenüber Flüchtlingen an der Grenze hätten. Auch könnten Flüchtlingsprobleme nicht angemessen mit rechtlichen Mitteln gelöst werden ${ }^{3}$. Diese Ansicht greift jedoch zu kurz. Denn das in Art. 33 I GFK geregelte Refoulement-Verbot erfordert irgendeine Form des Feststellungsverfahrens, um jene Personen identifizieren zu können, die den aus dieser Norm folgenden Rechtsschutz beanspruchen können ${ }^{32}$. Feststellungsverfahren sind demnach erforderlich, um die Gefahr der Verletzung des Prinzips des Non-Refoulements ausschlicßen zu können ${ }^{33}$. Andererseits beansprucht dieses Prinzip unabhängig von der formalen Prüfung des Asylbegehrens Geltung, ist also deklaratorischer Natur ${ }^{34}$. Dem Art. 33 I GFK wohnt damit immanent ein vorläufiges Aufenthaltsrecht inne ${ }^{35}$, um zu prüfen, ob der Asylsuchende sich auf die Schutzwirkung dieser Norm berufen kann.

27 Manuel R. Garcia-Mora, Internarional Law' and Asylum as a Human Right, Washington D.C. 1956, S. so.

28 Nemiah Robinson, Convention retating to the Status of Refugees, New York r953, S. 163; Atle GrahlMadsen, The Status of Refugees in International Law, Bd. 2, Leyden I972, S. 94; Otto Kimminich, Der inecrnationale Rechtsstatus der Fluchrlinge, Koln u. a. 1962, S. 327.

29 Guy S. Goodwin-Gill, The Refugee in International Law, Oxford 1983, S. 105; Paul Wieis, The International Protection of Refugecs, in: A Y'IL 1954, 199; James Crawford/Patricia Hyndman, Three Heresies in the Application of the Refugee Convention, in: IJRL 1989, 157; Walter Kalin, Das Prinzip des NonRefoulement, Bern u. a., 1982, S. 10s ff.; Kay Hailbronner, Das Refoulement-Verbot und die humanitaren Flüchtlinge, in: ZAR 1987, 5; a. M. Ernst Reichler, Das staatliche Asylrecht nim Rahmen des Vólkerrechts $\alpha$, Berlin 1987, S. 4 1.

30 Guy S. Goodwin-Gill, a. a. O, S. 97 ff.; Acle Grahl-Madsen, Identifying the World's Refugees, in: AAPSS 1983, 13; Prakash S. Sinha, Asylum and International I.aw, The Haguc i 971 , S. I60; Walter Kalin, a. a. O., 72; Paul Weis, a. a. O., S. 199; Robert C. Sexton, Policical Refugees, Non-Refoulement and Stute Practice: A Comparative Study, in: Vanderbuilt JTL 1985, 737; Patricia Hyndman, Refugees under International Law, I: The Australian LJ 1986, is3. Vgl. auch UNHCR Bericht F/1989/64, Nr. 24.

31 Kay Halbronner, a.a. O., S.6. Vgl. auch Kay Hailbronner, The Right to Asylum and the Furure of Asylum Procedures in the European Community, in: IJRL $1990,358$.

32 Guy S. Goodwin-Gill, The Principle of Access to National Procedures, 1984, S. 2 (unveroffentlichtes Manuskript).

33 UNHCR, Note on Intcrnational Protection, A/AC.96/694 v. 3.8.1987, Nr. 28.

34 Gilbert Jaeger, Status and International Protection of Refugees, International Institure on Human Rights 1978, Nr. 52; Guy S. Goodwin-Gill, The Refugee in International Law, a. .L O., S. 73.

3s Patricia Hyndman, a. a. O., S. 153; Deborah Perluss/Joan F. Hartman, Temporary Refugec: Energence of a Customary Norm, in: Virginia JIL 1986, 599; Robert C. Sexton, a. a. O., S. 730; Guy S. Goodwin* Gill, a. a.O.; Walter Kälin, a. a.O., S. 86; David Kennedy, International Refugee Protection, HRQ 1986,67 . 
Dementsprechend hat das durch 43 Staaten gebildete Exckutivkomitee des Programms des UNHCR in den letzten Jahren Empfehlungen zur Ausgestaltung der Asylverfahren verabschiedet. Zwar haben derartige Empfehlungen keine rechtliche Verbindlichkeit. Sic sind jedoch Teil des völkerrechtlichen Standards im Flüchtlingsrecht mit normativer Bedeutung ${ }^{36}$ und daher Richtlinien zur Ausgestaltung der Asylverfahren. $\mathrm{Zu}$ nennen sind hier insbesondere die Empfehlungen Nrn.8 (XXVIII) und zo (XXXIV) zur Verfahrensgestaltung sorvie Nr. I9 (XXXI), die die Staaten auffordert, bei Massenflüchtlingsproblemen zumindest vorübergehend Aufnahme zu gewähren. Für den europäischen Bereich ist auf die Empfehlungen Nrn. R (8r) I 6 und R (84) I des Ministerkomitees des Europarates hinzuweisen. Auch diese enthalten Schlüsselelemente zur Ausgestaltung dcr nationalen Asylverfahren. Im übrigen ist die Zahl dor Staaten, die Feststellungsverfahren einrichten, im Steigen begriffen ${ }^{37}$.

Zwar wird behaupter, daß selbst in europäischen Staaten häufig kein effektiver Schutz gegen Zurückweisungen gewährt werde ${ }^{3}$. Aus rechtlicher Sicht ist jedoch der Schluß vom Sein auf das Sollen untersagt. Vielmehr ist festzuhalten, daß sämtliche EG-Staaten Vertragsstaaten der GFK sind und damit das Refoulement-Verbot von Art. 33 I GFK zu beachten haben. Die UNHCR-Empfehlung Nr. 8 sorvie Europarats-Empfehlung Nr. R (81) 16 schreiben im übrigen ausdrücklich vor, daß die Staaten klare Anweisungen an die Grenzbehörden erlassen sollen, die sicherstellen, daß die Entscheidung über den Zugang zum Asylverfahren von der zuständigen Feststellungsbehörde getroffen wird. In nahezu allen europäischen Staaten sind jedoch Regelungen eingeführt worden, die den Zugang zum Asylverfahren in bestimmten Fällen ausschließen. Und auch die Bundesrepublik mißachtet internationale Standards, da weder $\$ 9$ I 2 AsylVfG a.F. noch $\$ 18$ II AsylVfG n. F. eine verwaltungsinterne Beteiligung der zuständigen Asylbehörde bei Zurückweisungen von Asylsuchenden vorschreiben. Das zentrale Problem des Rechtsschutzes gegen Zurückweisungen sind also die in vielen Staaten vorgeschalteten Zulässigkeitsverfahren.

\subsubsection{Zulässigkeitsverfabren}

UNHCR-Empfehlung Nr. 30 behandelt die Frage der offersichtlich unbegründeten Asylanträge und stellt für deren Behandlung bestimmte Mindeststandards auf. Danach soll auch in diesen Fällen die Entscheidung und die persönliche Anhörung durch die zuständige Asylbehörde getroffen werden. Gegen die negative Entscheidung soll eine Beschwerdemöglichkeit und Aufenthaltsrecht für die Dauer des Beschwerdeverfahrens gewährleistet werden. Eine zweite Gruppe der Zulässigkeitsverfahren (admissibility-procedure) bilden Fälle der Durchreise durch Drittstaaten ("safe host country«). UNHCR-Empfehlungen zur verfahrensrechtichen Behandlung dieser Fallgruppe gibt es nicht. Im EG-Bereich geben die Einwanderungsminister der Frage der Angleichung der unterschiedlichen Zulässigkeitsverfahren hohe Priorität. Ein Überblick über die Staatenpraxis verdeutlicht, daß diese Priorität gerechtfertigt ist.

36 Jerzy Sztucki, The Conclusions on the International Protection of Refugees adopted by the Executive Committee of the UNHCR Programme, IJRL 1989, 300.

37 UNHCR-Bericht E/1988/s3 spricht von uber so Staaten mit Feststellungsverfahren. Dagegen erwahnte der UNHCR-Bericht E/1987/s6 nahezu go Stuatun, wahrend UNHCR-Berıcht E/1986/ss lediglich 45 Staaten und UNHCR Note on International Protection A/AC.96/660 (1985) erst 40 Staaten auffuhrt. In den Berichten seit 1989 fehlen entsprechende Angaben

38 Kay Hailbronner, Das Refoulement-Verbot und die hum artaren Fluchelinge, in: ZAR 1987, 6. 
In Belgien wird seit r. Februar 1988 aufgrund einer Gesetzesändcrung vom i 4. Juli 1987 u. a. bei "Gefahren für die öffentliche Ordnung oder nationale Sicherheit«, bei "offensichtlich unbegründeten Anträgen", in Fällen, in denen der Asylsuchende in den letzten zehn Jahren ausgewiesen worden ist, sowie in den Fällen, in denen er sich vor seiner Einreise länger als drei Monate in einem Drittstaat aufgehalten hat, der Zugang untersagt. Hinzugekommen ist 1991 die sdoppelte-fünf-Prozent-Regelung «, derzufolge der Zugang versagt wird, wenn der Asylsuchende aus cinem Staat kommt, aus dem im Jahr zuvor mehr als fünf Prozent aller Asylsuchenden kamen. Weiterhin ist gefordert, daß weniger als fünf Prozent der Asylsuchenden dieses Landes im Jahr zuvor anerkannt worden sein nü̈ssen. Der Asylsuchende wird nicht über seine Rechte informiert, die Anhörung erfolgt durch die Grenzbehörde. Die Beweislast oblicgt dem Antragstellcr. Gegen die Zurückweisung kann Beschwerde beim Justizminister mit aufschiebender Wirkung eingelegt werden. Bestätigt der Minister nach Einholung der Zustimmung der zuständigen Asylbehörde die grenzbehördliche Entscheidung, wird kein Rechtsschutz gewährt und die Zurückweisung vollzogen. Diese Überprüfung ist binnen 24 Stunden, in zweifelhaften Fällen innerhalb von drei Tagen durchzuführen ${ }^{39}$. Nur in den Fällen, in denen Minister und Asylbehörde unterschiedlicher Ansicht sind, kann gerichtlicher Rechtsschutz beantragt werden. Das Gcricht hat innerhalb von fünfzehn Tagen zu entscheiden ${ }^{40}$.

In Dänemark wird seit Oktober 1986 durch die Grenzbehörde ein Verfahren praktiziert, demzufolge Aufenthalte in Drittstaaten zur Versagung des Zugangs führen. Insoweit wird jeglicher $Z$ wischenaufenthalt unabhängig von seiner Länge und seinem Charakter als ausreichend angesehen. Die Anhörung wird von der Grenzpolizei vorgenommen. Die Entscheidung trifft die für die Behandlung von Asylgesuchen zuständige Abteilung für Ausländer im Justizministcrium. Gegen deren Entscheidung kann Beschwerde beim Justizminister eingelegt werden. Dicse hat jedoch keine aufschiebende Wirkung. Ein besonderes Verfahren für »offensichtlich unbegründete Anträgc " wird nach der ersten Sachentscheidung durchgeführt (s. unten).

In Frankreich wird der Zugang zum Asylverfahren versagt, wenn der Asylsuchende bereits effektiven Schutz in einem Drittstaat gefunden hat. Diese Regelung wird auch dann angewandt, wenn es vernünftige Gründe für die Annahme gibt, daß der Betreffende im Transitstaat in zumutbarer Weise hätte Schutz, beantragen können ${ }^{4}$. Außerdem wird bei "offensichtlich unbegründeten Asylbegehren « der Zugang verweigert. Während dic Grenzbehörde die Anhörung durchführt, trifft der Innenminister nach Konsultation mit dem Außenministerium die Entscheidung. Letzteres beteiligt seit Oktober r991 die zuständigc Asylbehörde bei der Abfassung seiner Stellungnahme. Regelmäßig dauert dieses Verfahren nicht länger als eine Woche. Besondere Regelungen für die Gewährung von Rechtsschutz besıchen nicht. Seit Februar 1990 wird auch in Italien ein besonderes Zulassungsverfahren durchgeführt und der Zugang zum Asylverfahren versagt, wenn der Antragstellcr über einen verfolgungsfreien Drittstaat eingereist ist und sich dort "einige Zeit " ("trascorso un periodo di soggiorno «) aufgehalten hat. Der bloßc Transitaufenthalt fällt jedoch

39 Wissenschaftlicher Dienst des Deutschen Bundestages, Asylrecht unter besonderer Berucksichtigung des Verfahrensrechts in is europaischen Landern und in den USA, WF III-131/90, 21. 2. 1991, S.11. Im folgenden: Wissenschaftlicher Dienst.

40 R. Dedecker, The Right of Asylum in Europe. Some Proposals on Accelerated Procedures for the Twelfe Member States. Working Document. Prepared with the Support of UNHCR, Brussel 1992, S. 11. Nach amnesty international, Europe. Human Rights and the need for a fair asylum policy, EUR os/03/9l, S. 17 , ist dagegen die Beschwerde an die zuständige Asylbehorde zu richten und behält sich der Justizminister ungeachtet deren Entscheidung den Sofortvollzug vor.

4 I R. Dedecker, a. a. O., S. 19; die Praxis ist jedoch nucht klar gercgelt, s. Wissenschaftlıcher Dienst, a. a. O., S. 28 . 
nicht unter diesen Begrifft². Außerdem wird bei Vorliegen der Ausschlußgründe nach Art. rf GFK (Kriegsverbrechen, schweres nicht politisches Verbrechen) der Zugang versagt. Die Entscheidung trifft die Grenzbehörde nach persönlicher Anhörung ohne Beteiligung der zuständigen Asylbehörde. Zwar sieht das Gesetz cine Beschwerdemöglichkeit vor. Doch erfolgt hierüber keine Bclehrung und wird daher regelmäßig die Zurückwcisung unmittelbar vollzogen ${ }^{43}$.

In den Niederlanden wird Asylsuchenden, die über den Flughafen Schiphol einreisen, der Zugang verwehrt, wenn sie in einem Drittstaat sicher vor Verfolgung waren oder ihr Antrag "offensichtlich unbegründet" ist ${ }^{44}$. Kann der Bctreffende glaubhaft machen, daß er in den betreffenden Drittstaaten lediglich Transitreisender war, wird der Zugang eröffnet. Maßgeblich ist insoweit die Länge des Aufenthaltes. Die Beweislast obliegt dem Asylsuchenden. Die Anhörung crfolgt durch die Grenzbehörde, während der Justizminister die Entscheidung trifft. Hiergegen ist mit aufschiebender Wirkung Beschwerde zum Präsidenten des Bczirksgerichtes eröffnet. Das Verfahren dauert regelmäßig eine Woche bzw. sechs Wochen in Beschwerdefällcn'ss. Während dieses Verfahrens bleibt der Betreffende im grenzbehördlichen Gewahrsam ${ }^{46}$. Zu erwähnen ist noch, daß im Vereinigten Königreich in Fällen anderweitiger Sicherheit in Drittstaaten ein beschleunigtes Verfahren bestcht und Rechtsschutz für illegal eingereiste Asylsuchende gegen Zurückweisungen nicht gewälırt wird. Mir Blick auf die Frage der Verfolgungssicherheit in Drittstaaten ist die Rechtslage der Bundesrepublik vergleichbar mit den aufgezeigten Verfahren. Seit 1987 wird dic Zurückweisungspraxis insowcit jedoch äußerst restriktiv gehandhabt. Die Kategorie der "offensichtlich unbegründeten Asylbegehren " gewinnt wie in Dänemark erst nach der ersten Sachentscheidung besondere verfahrensrechtliche Bedeutung. In allen anderen Staaten der EG ist kein besonderes Zulässigkeitsverfahren dem eigentlichen Asylverfahren vorgeschaltet worden.

Das zentrale Problem des Rechtsschutzes gegen Zurückweisungen ist also die effektive Ausgestaltung der Zulässigkeits- und Schnellverfahren. Deren Gegenstand bilden drei Fallgruppen: Sonderverfahren für "offensichtlich unbegründete Anträge * sind bereits in viclen Staaten eingerichtet worden und werden aufgrund der Bemühungen der Einwanderungsminister bald zum allgemeinen Standardprogramm zählen. Das Konzept des "sicheren Staates" hat zweifache Bedeutung: Es dicnt einerseits zur Bestimmung des "sicheren Erstaufnahmelandes«. Während einige Staaten eine gewisse Dauer des Transitaufenthaltes voraussetzen (Belgien, Bundesrepublik, Frankreich, Italien und die Niederlande), läßt z. B. Dänemark den bloßen Transitaufenthalt genügen. In den Nicderlanden wird zudem stcts erst der Flüchtlingsstatus geprüft, bevor das "sichere "Erstaufnahmeland bcstimmt wird. Die zweite Funktion besteht in der Bestimmung des sog. sicheren Herkunttsstaates. Ausgehend von der schweizerischen Praxis hat sich bereits eine Arbeitsgruppe des Europarates mit einer Diskussionsvorlage des deutschen Mitglieds befa $\mathrm{At}^{47}$; und auch der UNHCR hat insoweit ein Konzept entwickelt, demzufolge Antragstellern aus wsicheren Herkunftsstaaten " zwar Zugang zum Verfahren gewährt werden solle, ihnen jedoch eine widerlcgbare Regelvermutung entgegengehalten werden könne $e^{48}$.

42 Bruno Nascimbene, The Albanians in Italy, IJRL 1992, 715; Wisscnschafclicher Dienst, a. a. O. S. SI.

43 ai, a. a. O., S. 14.

44 R. Dedecker, a.a. O., S. 40.

4 S. Dedecker, a. a. O., S. 4 t.

${ }_{4} 6$ Wissenschaftlicher Dienst, a. a. O., S. 59: maximal 30 Tage

47 Kay Hailbronner, The concept of safe country" and expedient asylun procedures, CAHAR (91) 2, 4. 9. 1991 .

48 UNHCR, Background Note on the Safe Country Concept and Refugee Status, EC/SCP/68, 26.7. 1991, Rdn. 7 u. 17; UNHCR, Working Document, Current Asylum Issues, Harmonization in Europe, Marz 
UNHCR-Empfehlung Nr. 8 nennt als Mindestanforderungen an das Feststellungsverfahren die Festlegung einer eindeutigen Zuständigkeit einer Behörde zur Prüfung und Entscheidung über das Asylgesuch in der ersten Insranz. Dies sollte nach Möglichkeit eine zentrale Bebörde sein. Außerdem sollte der Asylsuchende über seine Verfahrensrechte betehrt und sollten ihm die erforderlichen Hilfestcllungen, insbesondere die Dienste eincs sachkundigen Dolmetschers, garantiert werden. Überdies schreibt Empfehlung Nr. 30 mit Blick auf woffensichtlich unbegründete Anträge « ausdrücklich fest, daß der Asylsuchende durch einen qualifizierten Beamten - nach Möglichkeit durch einen Bediensteten der zentralen Feststellungsbehörde - ausführlich persönlich angebört werden sollte. Fmpfehlung Nr.R (81) 16 des Europarates empfichlt ebenfalls die sachliche Zuständigkeit einer zentralen Behörde. Des weiteren wird die Belehrungspflicht und das persönliche Anhörungsrecht unter Assistenz eines Dolmetschers vorgeschlagcn. Die Einrichtung einer zentralen Asylbehörde sowie das persönliche Anhörungsrecht des Asylsuchenden durch diesc Behörde sind demnach Schlüsselelemente des internationalen Rechtsschutzes.

Die überwiegende Mehrheit der europäischen Staaten, insbesondere die der EG, tragen diesen Mindestgrundsätzen im wesentlichen Rechnung. In Belgien war bis 1987 das Außenministerium für die Sachentscheidung in Asylverfahren zuständig. Durch ministericllen Erlaß von 1954 war diese Zuständigkeit auf den lokalen Vertreter des UNHCR delegiert worden ${ }^{49}$. Seit I. Februar 1988 entscheidet über alle Asylanträge nach unmittclbarer persönlicher Anhörung in voller Unabhängigkeit ${ }^{\text {so }}$ eine Bchörde (Commissaire Generale aux Refugies et aux Apatrides - CGRA). In der Bundesrepublik ist das Bundesamt für die Anerkennung ausländischer Flüchtlinge allein zuständige Asylbehörde $\left(\mathbb{S}_{4}\right.$ AsylVfG alt; $\$ s$ AsylVfG neu). Der Sachentschcider ist nach dem Gesetz weisungsunabhängig ( $\$ 4$ III I AsylVfG alt; $\mathbb{S}$ II I AsylVfG neu). Es besteht persönliches Anhörungsrecht durch das Bundesamt $(\mathbb{1}$ I 2 I 2 AsylVfG alt; $\mathbb{2} 25$ IV, V AsylVfG neu). In Dänemark ist dic Abteilung für Ausländer im Justizministerium (Direktoratet for Udlaendinge) zuständige Feststellungsbehörde mit unabhängiger Entscheidungskompetenz. Diese Abteilung führt jedoch nicht die Anhörung durch, sondern entscheidet auf Grundlage des polizeilichen Protokolls der Anhörung.

In Frankreich ist seit 1952 einc zentrale Behörde im Dienstbereich des Außenministeriums (Office francais de protection des refugies et apatrides - OFPRA) für die Prüfung und Entscheidung über Asylanträge zuständig. Bedienstete von OFPRA leiten die Untersuchungen in relativer Unabhängigkcit und hören in ca. 40\% der Fälle Asylsuchende persönlich an. Die Entscheidung selbst trifft der Direktor von OFPRA. Er wird durch den OFPRA-Rat (Conseil de l'Office) in grundsätzlichen Politikfragen beraten. Ministerielle Erlasse von 1977 haben in Griechenland die Ausländerabteilung des Nationalen Sicherheitsdienstes (Ministerium für öffentliche Ordnung) zur allein zuständigen Asylbehörde bestimmt. Ein Bediensteter dieser Abteilung führt die persönliche Anhörung durch. Irland hat kein besonderes Asylverfahren eingerichtet. Dic Anhörung wird durch die Ausländerbehörde durchgeführt. Das Justizministerium trifft unter Beteiligung des Außenministeriums die Sachentscheidung. Eine Beratung wird nicht gewährt. Weder die Beamten der unte-

1992, S. 4 f. Dagegen will der CDU/CSU-Gesetzentwurf zur Anderung von Art. 16 II 2 GG auch den Z.ugang zum Verfahren ganzlich unterbinden (BT-Drs. 12/21/2).

$49 \mathrm{~J}$. C.ls/G. I.oescher, The Refugee Determination Procedurc in Belgium and the role of the UNHCR, in: Refugees in the Age of Total War, A. Bramwell (Hrsg.), Oxford 1987, S.6; Council of Europe, Draft Report on living and working conditions of refugecs, AS/PR (36) 1, 19.11. 1984, S. 3.

so Wissensehafelicher Diense, a. .. O., S. 13. 
ren Behörde noch die des Ministeriums haben die erforderlichen Rechts- und Länderkenntnisse. Italien hat I 990 den lediglich auf europäische Flüchtlinge bezogenen Vorbehalt (Art. I B ra GFK) aufgehoben. Nunmehr werden alle Asylverfahren durch die im März 1991 eingerichtcte Commissione Centrale behandelt. Vorher war seit 1952 die schwerfällige, zahlreiche Ministerien beteiligende Gemeinsame Zulassungskommission zuständig. Die neu eingerichtete Kommission hört den Asylsuchenden an und trifft die Sachentscheidung.

Ebenso wic Irland hat Luxemburg kein Asylverfahren eingeführt. Das Ordnungsamt hört den Antragstellcr an, das Außenministerium trifft nach Konsultation mit dem Justizministerium die Entscheidung. In den Niederlanden ist die Flüchtlingsabteilung des Justizministcriums allein zuständige Asylbehörde. Ein Kontaktbeamter dieser Abtcilung leitet das Anhörungsverfahren. Auf Grundlage des Anhörungsprotokolles wird anschließend im Ministerium die Entscheidung getroffen. Wird nicht innerhalb von sechs Monaten entschieden, gilt der Antrag als abgeichnt. Seit 1987 wird bei »offensichtlich unbegründeten Anträgen « binnen Wochenfrist die Sachentscheidung getroffen'". Zuständige Asylbehörde in Portugal ist die aus Vertretern von sechs Ministerien bestehende Beratende Kommission (Commissao Consultativa para os Refugiados). Der Antrag ist innerhalb von 60 Tagen nach der Einreise zu stellen. Diese Kommission gibt auf der Grundlage der ausländerbehördlichen Anhörung eine Stellungnahme an das Innenministerium ab, welches die Entscheidung trifft. In Spanien ermittelt das Gencralkommissariat für Meldcangelegenheiten durch persönliche Anhörung den Sachverhalt und leitet den Vorgang einem interministeriellen Ausschuß zu. Sind Ausschlußgründe nach Art. x F GFK gegeben, entscheidet das Innenministerium über die Zulassung ${ }^{52}$. Auf Grundlage der Stellungnahme des Ausschusses entscheidet der Innenminister, wenn er zustimmt, andernfalls der Ministerrat. In Vereinigten Königreich ist dic Flüchtlingsabteilung des Innenministeriums allein zuständige Feststellungsbehörde. Die Anhörung führt die örtliche Ausländeroder Grenzbehörde durch. War der Asylsuchende nicht lediglich auf der Durchreise in einem sicheren Drittland ${ }^{33}$, wird der Asylantrag durch das Innenministerium unverzüglich - während des grenzbehördlichen Gewahrsams - abgelchnt. Auf Veranlassung des Ministeriums kann erneut angehört werden. Falls der Antrag abgelehnt werden soll und der Antragsteller keinen Rechtsbeistand hat, hat das Ministerium die Stellungnahme von UKIAS (United Kingdom Immigrant Advisory Service) einzuholen ${ }^{54}$. Besteht keine Übereinstimmung, hat der Minister die Sachentscheidung zu treffen.

Festzuhalten ist damit, daß in allen Staaten eine zentrale Behörde dic Sachentscheidung in Asylverfahren trifft, jedoch nur in wenigen Fällen diese Bchörde auch die pcrsönliche Anhörung leitet (Bundesrepublik, teilweisc Frankreich, mit Einschränkungen die Niederlande). In allen anderen Fällen führt die lokale Polizei-, Grenzoder Ordnungsbehörde die Anhörung durch und entscheidet die zuständige Asylbehörde nach Aktenlage. Gerade in Asylverfahren, in denen der Glaubwürdigkeitsprüfung überragende Bedeutung zukommt, ist dieses verfahrensrechtliche Defizit nicht hinnehmbar.

S1 Wissenschaftlicher Dienst, a. a. O., S.61.

s2 Wissenschaftlicher Dienst, a. a. O., S. gs.

53 Wissenschaftlicher Dienst, a. a. O., S. 41 .

54 Dieses Verfahren war zwischen 1988 und 1991 ausgesetzt worden. 
Die Frage des Überprüfungsverfahrens, insbesondere die rechtliche Natur der Beschwerdeinstanz, ist eines der sensibelsten Probleme bei der europäischen Harmonisierung der nationalen Asylverfahren. Von der sachgerechten Lösung dieser Frage hängt aus verfassungspolitischer Sicht dic volle Teilnahme der Bundesrepublik an den bestehenden Abkommen ab. UNHCR-Empfehlung Nr. 8 empfiehlt den Staaten, erfolglosen Asylsuchenden eine angemessene Frist einzuräumen, innerhalb deren sie eine formelle Überprüfung der Entscheidung beantragen können. Einen identischen Vorschlag enthält Empfehlung Nr. 30. Ausdrücklich wird die Notwendigkeit des Abschiebungsschutzes für die Dauer des Überprüfungsverfahrens in beiden Empfehlungen betont. Die Beschwerdemöglichkeit und das damit zusammenhängende Verbleibsrecht werden auch in der Empfehlung Nr. R (8I) 16 unterstrichen. Weitere Schlüsselelemente des internationalen Rechtsschutzes für Flüchtlinge sind demnach das Recht auf Überprüfung negativer Statusentscheidungen sowie der Abschiebungsschutz bis zum endgültigen Verfahrensabschluß.

Problematisch erscheint die Lösung des Rechtscharakters des Überprüfungsverfahrens. Nach Empfehlung Nr. 8 ist die Überprüfung durch dieselbe oder eine andere Behörde, sei es eine Verwaltungsbehörde oder ein Gericht, zulässig. Insoweit verweist die Empfehlung aber ausdrücklich auf das im jeweiligen Einzelfall vorherrschende Rechtssystem. Nach internationalem Stand sollcn Überprüfungen negativer Asylentscheidungen also nicht anders als andere gegen Behördenentscheidungen gerichtete Beschwerdcverfahren geregelt werden. Empfehlung Nr. R (81) r 6 nennt insoweit eine höhere Verwaltungsbehörde oder das gesetzlich vorgeschene Gericht. Nach Art. 8 der Allgemeinen Erklärung der Menschenrechte soll jedermann einen wirksamen Anspruch auf wirksamen Rechtsschutz vor den zuständigen innerstaatlichen Gerichten gegen Verwaltungsentschcidungen haben. Art. I 3 der Europäischen Menschenrechtskonvention (EMRK) spricht dagegen lediglich vom Recht auf eine wirksame Beschwerde bei einer nationalen Instanz.

Art. 13 EMRK setzt zunächst cinc Konventionsverletzung voraus, was in Asylfällen häufig bei Gefahren drohender Folter (Art. 3 EMRK) anzunehmen ist, aber bei anderen Verfolgungstatbeständen nicht zwingend ist. Andererseits erfordert Art. 13 EMRK nicht die vorherige Fcststellung der Konventionsvcrletzung durch den Gerichtshof für Menschenrechte (EGMR). Vielmchr soll mit dieser Norm dem einzelnen die Durchsetzung der Konventionsrechte im innerstaatlichen System ermöglicht werden"s. Der geforderte wirksame Rechtsschut< ("effective remedy ") ist aber nach dem Wortlaut von Art. I 3 und der Rechtsprechung des Gerichtshofes weniger als „gerichtlicher Rechtsschutz «. Wesentliche Voraussetzung eines wirksamen Rechtsschutzes ist nach dem Gerichtshof jedoch, daß die angerufene Instanz die angegriffenc Entschcidung überprüfen und aufheben kann. Kann sie lediglich Empfehlungen abgeben, genügt dies nicht ${ }^{56}$. Darüber hinaus fordert Art. ${ }_{3}$ EMRK eine inhaltliche Überprüfung der Verwaltungsentscheidung im Beschwcrdcverfahren ${ }^{57}$. Sofern gerichtlicher Rechtsschutz garantiert wird, hat das Gcricht den Vollzug der Verwaltungsentscheidung zu untersagen, wenn die angegriffene Maßnahme nicht rechtmäBig ist, rationalen Kriterien nicht genügt oder Verfahrensfehler enthält ${ }^{58}$.

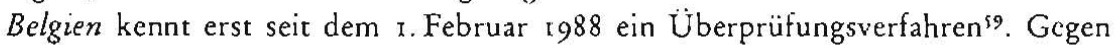

5s Frowein/Peukert, EMRK-Kommentar, Rdn. 2 zu Art. 13.

s6 Frowein/Peukert, a. a. O., Rdn. 3 zu Art. 13.

57 EGMR, Soering gegen Vereinigtes Konigreich, HRLJ 1990, 335 (368).

58 EGHR, a. a. O.; EGHR, Vlvarajah gegen Vereingtes Kongreich, HRLJ 1991, 432 (445).

s9 Zum fruheren Verfahren s. J. Cels/G. Loescher, a. 3. O., S. 7. 
negative Statusentscheidungen ist innerhalb von fünfzehn $\operatorname{Tagen}^{60}$ Beschwerde bei der Commission Permanente des Recours einzulegen. Dieses Organ besteht aus dem Vorsitzenden, dem Beisitzer sowie dem Vertreter des UNHCR und wird als unabhängiges Verwaltungsgericht bezeichnet ${ }^{6 t}$. Die Beschwerde hat aufschiebende Wirkung. Das Gericht nimmt einc inhaltliche Überprüfung der angefochtenen Entscheidung vor. Gegen eine ablehnende Gerichtsentscheidung kann bei Fragcn rechtsgrundsätzlicher Bedeutung innerhalb von zwei Monaten, jedoch ohne aufschiebende Wirkung eine Entscheidung dcs Staatsgerichtshofes (Conseil d'Etat) beantragt werden. In der Bundesrepublik kann innerhalb von zwci Wochen Klagc bcim Verwaltungsgericht erhoben werden $(\$ 72$ I AsylVfG). Diese ist binnen Monatsfrist zu bcgründen ( $\$ 72$ II I AsylVfG) und hat aufschiebende Wirkung ( $\$ 73$ i.V.m. 38 AsylVfG). Gegen die Klageabweisung kann auf Antrag durch das Berufungsgericht Berufung zugelassen werden ( $\$ 76$ II AsylVfG). Weitere Rechtsbehelfe bleiben möglich.

In Dänemark entscheidet über Beschwerden der Refugee Appeals Board, welcher aus Vertretern von drei Ministerien, zwei Vertretern des Flüchtlingsrates, einem Vertreter der Anwaltskammer und einem Richter als Vorsitzendem bcsteht ${ }^{62}$. Mehrere Kammern mit jeweils sieben Mitgliedern sind eingerichtet worden. Dieses Organ wird allgemein als unabhängig und seine Entscheidungen als gerichtsähnlich angesehen. Die Beschwerde hat aufschiebende Wirkung. Hat das Ministerium den Antrag jedoch als "offensichtlich unbegründet " abgelehnt, ist deren Weitergeltung von der Stellungnahme des Flüchtlingsrates abhängig. Zu diesem Zwcck führt der Flüchtlingsrat in jedem Einzelfall eine persönliche Anhörung durch. Bestätigt er die Behördenentscheidung, wird die Abschiebung vollzogen. In etwa $20 \%$ der Fälle gibt der Flüchtlingsrat ein abweichendes Votum ab. In diesen Fällen wird das Beschwerdeverfahren durchgeführt. In »offenbar unbegründeten Fällen « kann überdies ein Dreierausschuß die Beschwerde als unzulässig abweisen ${ }^{63}$. Regelmäßig hört der Beschwerdeausschuß den Asylsuchenden an und überprüft inhaltlich. Seine Entscheidung ist nicht mehr anfechtbar. Der Betreffende kann den Ombudsmann anrufen.

Die zuständige Beschwerdeinstanz in Frankreich ist die aus zehn Kammern bestehende Commission des Recours des Refugies. Die Kammern bestehen jeweils aus einem Richter des Staatsrates als Vorsitzendem, dem Vertreter des OFPRA-Rates und einem Vertreter des UNHCR. Es wird als Vertvaltungsgericht bezeichnet ${ }^{64}$. Die Bcschwerde ist innerhalb eines Monats einzulegen. Der Suspensiveffekt ist geserzlich nicht geregelt, wird aber regelmäßig angeordnet ${ }^{6 s}$. Die Rechtsbehelfsfrist gilt zugleich als Begründungsfrist. Entscheidet OFPRA nicht binnen vier Monaten, gilt der Antrag als abgelehnt. Eine Rechtsbehelfsfrist besteht insoweit offensichtlich nicht ${ }^{66}$. Der Ausschuß hört zwar persönlich an, stellt jedoch keinen Dolmetscher zur Verfügung. Die extreme Kürze der Verhandlungen und die Art der Ermittlungen waren in der Vergangenheit wiederholt Gegenstand öffentlicher Kritik. Gegen ablehnende Entscheidungen des Ausschusses kann Beschwerde zum Staatsrat (Counseil d'Etat) binnen zwei Monaten, jedoch ohne aufschiebende Wirkung eingelegt werden. In

60 Wissenschaftlicher Dienst, a. a. O., S. 13, nennt cine Frust von 30 Tagen.

6. R. Dedecker, a.a.O., S. 12 ; ahnl. Wissenschaftlicher Dienst, a.a. O. Dort wird jedoch eine andere $\mathrm{Zu}$ sammensetzung genannt: Ein Richter, jewells ein Beamter des Außen- und Jusuzministeriums, ein Rechtsanwalt, UNHCR mit beratender Stımme.

62 R. Dedecker, a. a. O., S. 16 FN 7.

63 Wissenschaftlicher Dienst, a. a. O., S. 23.

64 R. Dedecker, a. a. O., S. 20; Wissenschaftlicher Dienst, a. a. O., S. 30

6s Wissenschaftlicher Dienst, a. a. O.

66 Wissenschaftlıcher Dienst, a.a. O. 
Griechenland wird lediglich ein verwaltungsinternes Überprüfungsverfahren gcwährleistet. Der Antragsteller kann binnen dreißig Tagen Bcschwerde mit aufschiebender Wirkung beim Minister für öffentliche Ordnung einlegen. Dieser entscheidet nach Konsultation eincs interministeriellen Beratungsgremiums. Der UNHCR-Vertreter hat Anwesenheits- und Anhörungsrecht. Weitere Rechtsbehelfe soll es nicht geben $^{67}$. In Irland ist keine Bcschwerdemöglichkeit vorgesehen ${ }^{68}$. In Italien entscheidet seit dem 28. Februar $1990^{69}$ das Tribunale Administrative Regionale, ein Verwaltungsgericht, über Beschwerden gegen ablehnende Statusentscheidungen. Diese haben aufschicbende Wirkung. Es werden jedoch nur Vcrfahrensfehler berücksichtigt. Die Sache wird im Fall eines derartigen Fehlers an die Commissione Centrale zurückverwiesen. Auch in Luxemburg wcrden lediglich Vcrfahrensfehler und Grundsatzfragen im Gerichtsverfahren berücksichtigt. Zuständig ist der Staatsrat. Die aufschiebende Wirkung der Klage kann beantragt werden, braucht jedoch regelmäßig wegen der kurzen Verfahrensdauer nicht angeordnet zu werden ${ }^{70}$.

In den Niederlanden entscheidet das Justizministerium nach Konsultation eines unabhängigen beratcnden Ausschusses über dic Beschwcrde. Regclmäßig akzeptiert der Minister dessen Stellungnahmc. Grundsätzlich hat die Beschwerde aufschiebende Wirkung. In Fällen, in denen das Ministerium jedoch vernünftige Zweifel an der Flüchtlingseigenschaft oder an der fehlenden Sicherheit im Drittstaat hat, kann es den Sofortvollzug anordnen. Gegen diese Anordnung kann gerichtlicher Rechtsschutz im Eilverfahren beantragt werden. Während dieses Verfahrens wird nicht abgeschoben". Nach Abschluß des Beschwerdeverfahrens kann der Staatsgerichtshof (Raad van State) angerufen werden. Seit 1990 hat eine Beschwerde keine aufschiebende Wirkung mehr. In Portugal kann die ablehnende Statusentscheidung innerhalb von einem Monat beim Oberverwaltungsgericht (Supremo Tribunal Administrativo) mit aufschiebender Wirkung angefochten werden. In Spanien kann gegen die Versagung des Zugangs zum Verfahren gerichtlicher Rechtsschutz beantragt werden. Wird der Asylstatus verweigert, ist der Rechtsweg ausgeschlossen. Gegen die Versagung des Flüchtlingsstatus kann aber das Verwaltungsgericht mit aufschiebender Wirkung angerufen werden ${ }^{72}$. Im Vereinigten Königreich kann gegen Entscheidungen des Innenministeriums binnen vierzehn Tagen Beschwerde beim Beschwerdcbeamten (Immigration Appeals Adjudicator) eingelegt werden. Dieser wird vom Innenminister ernannt. Ist der Aufenthalt des Asylsuchenden rechtmäßig, hat die Beschwerde aufschiebende Wirkung. Rcist der Asylsuchende dagegen ohne Visum ein, hat sein Rechtsbehelf keinen Suspensiveffekt. Es besteht persönliches Anhörungsrecht. Auf Zulassung kann gegen die ablehnende Entscheidung des Bcamten binnen zwei Wochen ein Rechtsbehelf beim Beschwerdegericht (Immigration Appeals Tribunal), einem aus drei Beamten bestehenden Ausschuß, eingelegt werden. Dieser berücksichtigt lediglich Grundsatzfragen oder schwcrwiegende Verfahrensfchler. Gegen die Entscheidung des Ausschusses kann nach Zulassung Klage beim Oberen Zivilgericht sowie abschließend beim Oberhaus erhoben werden.

Zusammenfassend ist festzuhalten, daß in der Mehrzahl der EG-Staaten unabhän-

67 Jedoch war der Oberste Verwaltungsgerichtshof wiederholt mit Asylverfahren befaßr. z. B. 1JRL 1992, $740 ;$ l]RL 1992, 741 .

68 Unklar ist, ob es gerichtlichen Rechtsschutz gegen Ausweisungen und Abschiebungen gibt, s. Wissenschaftlicher Dienst, a. a. O., S. 37.

69 Vorher gab es kein Beschwerdereche, Council of Europe, a. a. O., S. 9.

70 Wissenschafelicher Dienst, a. a. O., S. $\$ 7$.

71 Aldo Kuijer/Aleıdus Woltjer, Migration in Europe, NCB, 1991, S. 17

72 Wissenschaftlicher Dienst, a. a. O., S.96; a. M. R. Dedecker, a. a. O., S. 49: Unmittelbarer Rechtsweg zum Verwaltungsgericht bei Verweigerung des Fluchtlingsstatus. Bei Asylversagung ist dem Gerichtsverfahren ein ministerielles Ùberprufungsverfahren vorgeschaltet. 
gige Beschwerdeinstanzen (Dänemark, Niederlande, Vereinigtes Königrcich nach Zulassung) oder sogar Verwaltungsgerichte (Belgien, Bundesrepublik, Frankreich, Italien, Portugal und Spanien) negative Statusentscheidungen überprüfen. Nur in Irland ist keine Beschwerdemöglichkeit und in Griechenland nur eine verwaltungsinterne Überprüfungsinstanz eingerichtet worden. In einigen Staaten ist die gerichtliche Kontrolle auf rechtliche Aspekte beschränkt (Italien, Luxemburg und Vereinigtes Königreich). In aller Regel hat die Beschwerde aufschiebende Wirkung. In einigen Staaten stehen weitere Rechtsbehelfe, jedoch anders als in der Bundesrepublik ohne Suspensiveffekt (Belgien, Frankreich und Niederlande) zur Verfügung.

\subsection{Rechtsanspruch auf Zugang zum Verfahren}

Da die Rcgelung von Zulässigkeitsverfahren politische Priorität hat, ist das Recht auf Zugang zum Verfahren zu untersuchen. So hebt z. B. in der erwälmten Kommunikation die Kommission hervor, daß die Abschaffung der Binnenkontrollen bis zum 3r. Dezember 1992 insbesondere cin gemeinschaftliches Recht auf Asyl (S. r) voraussetze. Zuallererst sei nämlich das Asylrecht ein Recht und einc humanitäre Forderung (S. 2). Eines der Ziele der Dubliner Konvention sei es, das Entstehen von "refugees in orbit« zu verhindern. Daher sei mit der formalen Anerkennung des Rechts auf Asyl durch diese Konvention bereits ein wichtiger Schritt unternommen worden (S. 4). Es wäre in der Tat ein fundamentaler Fortschritt, würde ein völkerrechtlicher Vertrag das subjektive Recht auf Asyl verbindlich anerkennen. Daher ist die Kommission in ihrer politischen Wertung der Dubliner Konvention zu unterstützen. Der juristische Text trägt allerdings deren Behauptung nicht. Denn die Konvention regelt nur die Verpflichtung der Mitgliedsstaaten, jedes Asylgesuch zu behandeln (Art. 3 Abs. I). Weder wird - wie in den menschen rechtlichen Verträgen ein subjektiver Anspruch formuliert noch enthält die Konvention eine staatliche Verpflichtung, das subjektive Recht auf Zugang zum Asylverfahren im innerstaatlichen Recht zu regeln.

Ein weiteres unabdingbares Element bei der Gestaltung des europäischen Asylrechts ist daher dic rechtsverbindliche Regelung eines subjektiven Rechts auf Zugang zum Asylverfahren wenigstens in einem der Vertragsstaaten. Zu unterscheiden vom Rccht auf Zugang zum Verfahren (right to seek asylum.) ist das Recht auf Asyl (right to obtain asylum), welches in der Dubliner Konvention ebenfalls nicht anerkannt wird. Denn nach Art. 2 erncuern die Vertragsstaaten lediglich ihre Verpflichtungen nach der GFK und dem Protokoll von 1969. Beide Verträge enthalten jedoch nur Staatenverpflichtungen. In der Praxis aller westlichen Staaten ist mit der Gewährung des Flüchtlingsstatus jedoch in aller Regel ein sicheres Aufenthaltsrecht verbunden. Lcdiglich in den Vereinigten Staaten ist die Flüchtlingsanerkennung in das Ermessen der Einwanderungsbehörde gestellt. Deshalb kann für einen derart historisch, politisch und rechtlich miteinander verwobenen Bereich wie dem der EG die Forderung aufgestellt werden, daß das subjektive Recht auf Zugang zum Verfahren sowie auf Asyl vertraglich zu regeln ist. Es besteht insoweit Übereinstimmung, daß der Flüchtlingsbegriff nach Art. I A GFK alleinige Grundlage der materiellen Harmonisierung scin kann.

\subsubsection{Bestimmung des Erstaufnabmelandes}

Das subjektive Zugangsrecht setzt zuallererst eine Klärung des Prinzips des »sicheren « Drittstaates voraus. Wic crwähnt, ist bislang in aller Regel das Flüchtlingsproblern auf Kosten des Nachbar- oder des Trsttransitlandes gelöst worden. Die Schen- 
gener und EG-Regierungen haben seir Mitte der achtziger Jahre Bestrebungen unternommen, für ihren Bereich diesen Wildwuchs durch vertragliche Festlegung der Zuständigkeit eines einzigen Staates zu regeln. Auch im Europarat ist ein entsprechender Entwurf ausgearbcitet worden. Überdies wird überlegt, die Dubliner Konvention für andere Staaten offenzuhalten. Priorität hat jedoch vorerst die Ratifizierung der Konvention durch die EG-Staaten ${ }^{73}$. Dic Schengener Vertragsstaaten haben überdies am 29. Märı 1991 mit Polen ein Abkommen zur Rückübernahme von Asylsuchenden nach dem Schengener Muster unterzeichnet. Weitere Abkommen mit osteuropäischen Staaten, aber auch mit Österrcich und der Schweiz sollen folgen. Inzwischen werden auch mit den nordamerikanischen Staaten, ja selbst mit Marokko Verhandlungen zu diesem Zweck geführt.

Das erste Politikkonzept zielt damit auf die vertragliche Regelung des Erstaufnahmelandes. Vorherrschendes Problem ist hierbei die Notwendigkeit einer vorherigen prozessualen und materiellen Harmonisierung. Der Zugang zum Asylverfahren wird dabei im Grundsatz anerkannt, jedoch in den Fällen versagt, in denen ein anderer Vertragsstaat für die Behandlung des Antrags zuständig ist. Daneben wird derzeit eine Konzeption erarbeitet, welche die Zurückwcisung und damit die Verweigerung des Zuganges zum Verfahren auch dann zulassen soll, wenn zwar ein anderer Vertragsstaat originär zuständig wäre, dic Zurückweisung in einen als sicher angesehencn Erstaufnahmestaat außerhalb der Vertragsstaaten aber möglich erscheint. Ist also im ersten Fall ein Vertrag Grundlage für die Bestimmung des Erstaufnahmelandes, fehlt es hieran im zweiten Fall.

Grundlage für die vertragslose Zurückweisung ist ein Vertrag sclbst, nämlich Art. 3 Abs. 5 der Dubliner Konvention. Danach hat ein Vertragsstaat das Recht, den Asylsuchenden in einen Drittstaat zurückzuweisen. Zur Harmonisierung der insowcit unterschiedlichon Praxis schlagen die Einwanderungsminister vor, daß die Zurückweisung zulässig sein soll, wenn der Asylsuchende bei seiner Durchreise durch den Nichtvertragsstaat die Möglichkeit der Gewährung von Vcrfolgungsschurz gehabt habe und nach der Zurück weisung vor Verletzungen des Non-Refoulement Verbotes und vor Gefahren für Leib und Leben sicher sei. Durch die Zurückweisung in einen Nichevertragsstaat werde der originär zuständige Vertragsstaat von seiner Verpflichtung zur Behandlung des Asylgesuchs befreir ${ }^{74}$. Vorrang vor der vertraglich geregelten Verweisung eines Asylsuchenden an den zuständigen Staat soll demnach die Abdrängung des Flüchtlings an einen Nichtvertragsstaat, regelmäßig ein Land der sog. Dritten Welt, haben. Bemerkenswert ist, daß noch im Entwurf vom 3. April 1992 die konkrete Möglichkeit der Gewährung von Verfolgungsschutz im Drittstaat gefordert wurde ${ }^{7 s}$. Durch den Wegfall dieses Erfordernisses im Entwurf vom 9. April 1992 wird einer rein abstrakten, von den subjektiven Verhältnissen abstrahiercnden Betrachtungsweise der Vorzug gegeben. Die Einwanderungsminister wollen also die Tradition der Abdrängung der Flüchtlinge in außereuropäische Transitländer fortsetzen und handeln damit dem erklärten Ziel der Dubliner Konvention, nämlich das Phänomen von »refugees in orbit « zu beseitigen, zuwider.

Demgegenüber verlangt die Kommission in ihrer erwähnten Kommunikation, daß bei der Bestimmung des Erstaufnahmelandes die »legitimen Interessen « des Asylsuchenden Berücksichtigung finden (S. 16). Ausgangspunkt muß daher Art. 3 Abs. S der Dubliner Konvention sein. Danach darf die Zurückweisung in einen Drittstaat

73 Commission of the EC, a. a. O., S. 13

74 Groupe Ad Hoc Immigration, Definition et application harmonisee du principe du pays de premier accuell, SN 2086/92, WGI 106 I, 9.4.1992, S. 4.

75 SN $2074 / 92$ WGI $1049,3.4$. 1992, S. 2 l., s. 
nur unter Berücksichtigung der Verpflichtungen aus der GFK erfolgen. Zwar untersagt Art. 33 Abs. I GFK lediglich die Zurückweisung in den bchaupteten Verfolgerstaat. Nach Art. 31 Abs. 2 GFK begründet der vorübergehende Aufenthalt in einem Transitstaat jedoch noch keine Verpflichtung dieses Staates zur Rückübernahme. Vielmehr haben Transitstaaten nach Art. 3 Abs. 2 GFK dem Flüchtling eine angemessene Überlegungsfrist zu gewähren, bis es ihm gelungen ist, Aufnahme in einem anderen Land zu finden. Da die EG-Staaten bei der Anwendung von Art. 3 Abs. S der Dublincr Konvention an die GFK gebunden sind und diese nach Treu und Glauben anzuwenden haben (Art. 3x Abs. \& Wiener Vertragsrechtskonvention), dürfen sie allein aus dem bloßen Aufenthalt in einem Nichtvertragsstaat kcine Rücknahmeverpflichtung und damit korrespondierend Zurückweisungsbefugnisse ableiten. Die Intexpretation des Begriffs des Erstaufnahmelandes durch die Einwanderungsminister ist demnach mit Völkerrecht unvereinbar.

Die Empfehlung Nr. 22 (XXXII) des Exekutivkomitees des UNHCR legt Behandlungsstandards für Flüchtlinge, die lediglich vorübergehend Aufnahme erhalten, fest. Diese Empfehlung will den Staaten bei Massenfluchtbewegungen einige Hilfestellungen geben. Selbst wenn ein Transitstaat in Übereinstimmung mit dieser Empfehlung insbesondere das Diskriminierungsverbot und dic Menschenrechte beachtet sowie materielle Hilfestellungen gewährt, kann ein anderer Staat jedenfalls unter Berücksichtigung dieser Empfehlung einen Flüchtling nicht in diesen Transitstaat zurückweisen. Diese Empfehlung verlangt nämlich eine internationale Zusammenarbcit bei der Bewältigung von Massenfluchtbewegungen. Weder Art. 31 Abs. 2 GFK noch Empfehlung Nr. 22 erlauben daher eine Zurückweisungspraxis, die ausschließlich an den bloßen Transitaufenthalt anknüpft. Denn dieser Aufenthalt allein begründet noch keine Verpflichtung des betreffenden Staates zur Rückübernahme des Flüchtlings. Diese ist jedoch Voraussetzung, um von einem sicheren Drittstaat ausgehen zu können. Erst die konkrete Schutzgewährung durch einen Drittstaat und damit die Aussicht auf eine Dauerlösung ("durable asylum") oder wenigstens die konkrete Garantie auf Zulassung zum Asylverfahren läßt den Schluß auf ein effektiv sicheres Erstaufnabmeland zu.

Die Lösung liegt in der GFK selbst: Deren Erlöschensklauseln (Art. I C und E) verdeutlichen, daß Asylsuchende solange schutzbedürftig sind, wie ihnen kein dauerhafter Schutz gewährt worden ist ${ }^{76}$. Dicse Ansicht wird durch das Mandat des UNHCR, Flüchtlingen eine dauerhaftc Lösung entweder durch freiwillige Rückkehr, Integration in den Asylstaat oder Weiterwanderung in einen anderen Staat zu verschaffen, bekräftigt. Nach Empfehlung Nr. Is (XXX) des Exekucivkomitees des UNHCR soll Asyl nicht allein aus dem Grund versagt werden, weil es in eincm anderen Staat hätte beantragt werden können. Vielmehr sollen auch die Absichten des Flüchtlings hinsichtlich des erstrebten Asylstaates berücksichtigt werden. Mit dem Hinweis der Kommission auf die "legitimen Interessen der Asylsuchenden" wird dieser internationale Standard in Bczug genommen. Das bedeutet keine Anerkennung des Prinzips der freien Wahl des Asylstaates. Vielmehr erfordern Art. 33 Abs. I und 3 I Abs. 2 GFK die hinreichend sichere Feststellung, daß wegen des Aufenthaltes des Flüchtlings in einem anderen Staat dieser zur Rückübernahme verpflichtet ist. Diese Verpflichtung entsteht jedoch erst dann, wenn ihm dort dauerhafter Schutz gewährt wurde. Insoweit sind die glaubhaft gemachten Absichten des Asylsuchenden Beweisanzeichen in die eine oder andere Richtung. Versichert er glaubhaft, daß er im Transitstaat nicht Schutz beantragt hat, ist ihm dort auch nicht

76 Gilbert Jacger, a. a. O., Rdn. 69. 
der nach der GFK gebotene dauerhafte Schutz zuteil geworden und steht daher Art. 3 Abs. s der Dubliner Konvention einer Zurückweisung in diesen Staat entgegen.

\subsubsection{Rechtsanspruch auf Zugang zum Staatsgebiet}

Wichtigste Voraussetzung für den effektiven Gebrauch des Rechts auf Prüfung des Asylgesuchs ist die Zulassung zum Staatsgebiet selbst. Das Schengener Zusatzabkommen verpflichtet die Vertragsstaaten zur Einführung einer gemeinsamen Visapolitik (Art. 9). Ebenso sollen gemeinsame Regelungen zur Rückbeförderungspflicht (Art. 26 Abs. Ia) sowie zur Bestrafung von Beförderungsunternehmen (Art. 26 Abs. 2) geschaffen werden. Identische Bestimmungen sieht der EG-Entwurf einer

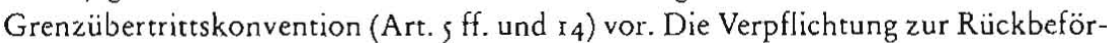
derung illegal Einreisender ist traditionelle Praxis in Westeuropa. Seit Mitte der achtziger Jahre haben zudem schrittweise Belgien, die Bundesrepublik, das Vereinigte Königreich und Dänemark finanzielle und strafrechtliche Sanktionen eingeführt, die den Zweck verfolgen, insbesondere Fluggesellschaften vor dem Abflug aus Transit- und Herkunftsstaaten zur peinlichen Kontrolle der Dokumente der Reisenden anzuhalten ${ }^{77}$. Inzwischen hat Frankreich ebenfalls Strafsanktionen gegenüber Beförderungsunternehmen eingeführt. Allerdings entfällt die Sanktion, wenn der Asylsuchende zum Asylverfahren zugelassen wird. UNHCR und nichtstaatliche Organisationen haben diesc Maßnahmen als Politik der Fluchtverhinderung kritisiert und schwerwiegende völkerrechtliche Bedenken hiergegen erhoben. Insbesondere wird eine Politik, die bewußt oder zumindest billigend in Kauf nimmt, daß Flüchtlinge an der Flucht gehindert werden, als unvereinbar mit der GFK bezeichnet. Denn deren Präambel will Flüchtlingen die Ausübung der Menschenrechte im großen Umfang gewährleisten. Zudem widerspricht es Treu und Glauben (Art. 3 I Abs. I Wiener Vertragsrechtskonvention), die Bestimmungen der GFK in einer diese Ausübung verhindernden Weise anzuwenden ${ }^{78}$.

Mit Blick auf Art. 16 Abs. 2 Satz 2 GG hat das Bundesverwaltungsgericht die deutschen Sanktionsbestimmung gegenüber Bcförderungsunternchmen für unvereinbar mit der Verfassung erklärt und deshalb diese Frage dem Bundesverfassungsgericht zur Entschcidung vorgelegt ${ }^{79}$. Derartige Bestimmungen ver- oder behinderten die durch Art. I6 Abs. 2 Satz 2 GG gewährlcistete Einreisemöglichkeit und seien deshalb wegen Verstoßes gegen den objektiven Wertgehalt dicses Grundrechtes nichtig. Unter Bezugnahme auf eine einschränkende Literaturmeinung hat das Gericht allerdings keinen völkerrechtlichen Verstoß erkennen können. Zwar umfasse Art. 33 Abs. I GFK auch das Zurückwcisungsverbot an der Grenze, es enthalte jedoch keine Aufnahmeverpflichtung. Diese Ansicht greift zu kurz. Das Beförderungsverbot verletzt das $\mathrm{Zu}$ rückweisungsverbot ${ }^{80}$. Art. 33 Abs. I GFK untersagt nämlich die Ausweisung oder Zurückweisung »auf irgendeine Weise « über die Grenzen des Verfolgerstaates. So sind z. B. nach der UNHCR-Empfehlung Nr. 53 (XXXIX) Asylsuchende, die als blinde

77 Überblick uber diese Praxıs: A. Cruz, Compatibility of carrier sanctions in four Community states with international civil aviation and human rights obligation, in: Meijers u. a., Schengen, Utrecht 199 I, S. 37 ff. Seit r 990 werden in Italien See- und Schiffahrtsunternehmen mit Geldbußen belegt, wenn sie illegal Einreisende nicht den Behorden melden (Wissenschaftlicher Dienst, a. a. O., S. 53). Außerhalb Europas haben Australien, Brasilien, Kanada, Neu-Seeland, Thailand und dic Vereınıgten Staaten derartige Sanktionen eingefubrt.

78 UNHCR, Sanctions against Transpors Companies, 1987; Erika Fullcr, Carrier Sanctions and International Law, IJRL. 1989,59 .

79 BVerwG, B. v. 14.4. 1992-BVerwG $1 \mathrm{C}_{48} 8.89-\mathrm{NVwZ}_{1992,682 .}$

80 Erika Feller, a.a. O., S. 58 ; dies., Transport Carriers and Refugce Protecuion, in: The Role of Airline Companies, Danish Refugee Council, 1988, S. .9. 
Passagiere auf See angetroffen werden, und Flüchtlinge in Booten gegen Zurückschiebung auf See durch Art. 33 Abs. I GFK geschütz ${ }^{8}$. Jegliche staatliche Maßnahme, dic dazu führt, daß ein Flüchtling gegen seinen Willen in das Gebiet des Verfolgerstaates gelangt, ist mithin nach dieser Norm untersagt. Wie dem Asylrecht ist dem Refoulementverbot auch eine verfahrensrcchtliche Vorwirkung immanent. Beförderungsverbote verhindern jedoch den Zugang zum Verfahren und sind auch deshalb völkerrechtlich nicht zulässig ${ }^{z_{2}}$. Das Völkerrecht verlangt nämlich effektive Verfahren und damit verfahrensrechtliche Vorkchrungen gegen die Verletzung des Refoulementverbotes $^{8}$. Der Hinweis des Bundesver waltungsgerichtes auf entgegenstehende Staatenpraxis vcrfängt nicht. Denn das Verbot des Refoulement mit seiner verfahrensrechtlichen Schutzwirkung hat nicht nur gewohnheitsrechtlichen Rang, sondern sogar den Charakter von jus $\operatorname{cogens}{ }^{8_{4}}$. Ein Abweichen von dieser Regcl ist folglich rechtlich ausgeschlossen.

Dem Bundesvcrfassungsgericht ist nunmehr der "schwarze Peter zugeschoben worden. Bestätigt es die Ansicht des Bundesverwaltungsgerichtes, ist die Gestaltung der europäischen Asylpolitik in einen wichtigen Teilbereich blockiert. Denn eine effektive Abwehr von Flüchtlingen setzt eine gemeinsame Politik aller EG-Staaten voraus. Dementsprechend erlauben die Abkommen in dieser Frage auch keine nationalen Vorbehalte. Unabhängig von der verfassungsrechtlichen Zulässigkeit verletzt das Beförderungsverbot zwingendes Völkerrecht und kann daher nach Art. 25 Satz 2 GG keine Geltung erlangen. Andererseits ist die Sanktionspolitik für dic Bundesrepublik nur von untergeordneter Bedcutung. Denn angesichts der offenen Grenzen zum Osten hat die Bundesregierung ein erhebliches Interesse an weiteren Abkommen mit östlichen Staaten zur Regelung der Rückübernahmeverpflichtungen. Dies wiederum wirft verfassungsrechtliche Fragen auf.

\section{Nationale Vorbehalte und europäische Harmonisierung}

Das Schengener Zusatzabkommen (Art. 29 Abs. 4) und die Dubliner Konvention (Art. 3 Abs. 4) erlauben einem Vertragsstaat nach seinem innerstaatlichen Recht ein Asylgesuch zu behandeln, obwohl ein anderer Vertragsstaat hierfür zuständig wäre. Die Kommission hat andererseits in der erwähnten Kommunikation unmißverständlich klargestelt, daß ein Recht zur mehrfachen Antragstellung in der EG nicht existieren sollte. Dies bedeute, daß kcin Staat von den erwähnten Vorbehalten Gebrauch machen sollte (S. ५). In den Niederlanden hat der Staatsrat demgegenüber in seiner Empfehlung vom 8. April $1999^{8}$ s der Regierung empfohlen, das Schengener Zusatzabkommen nicht zu ratifizieren. Unabdingbare Voraussetzung hierfür sei nämlich eine matericlle und prozessuale Harmonisierung des Asylrechts in den Vertragsstaaten. Da Mitgliedsstaaten der GFK die Verpflichtung zur Behandlung eines Asylgesuchs hätten, sei eine Übertragung der Verantwortung hierfür auf einen anderen Staat nur zu verantworten, wenn dieser ähnliche Standards anwende und ein faires Verfahren bereithalte. Die holländische Regierung ist $z$ war an diese Empfehlung nicht gebunden, hat jedoch Verhandlungen eingeleitet, um in einem gewissen Umfang die

81 UNHCR, Note on International Protection, A/AC. $96 / 728$, Nr. 18, 2.8. 1989.

82 H. Meiyers, Refugees in Western Europe, in: IJRL 1990, 434.

83 Guy S. Goodwin-Gill, The Determination of Refugee Status, in: The International Institute of Humanitarian Law, Ycarbook 1985, S. 57.

84 UNHCR-Bericht, E/1989/64, Nr. 29, 9. 5.1989.

$85 \mathrm{Nr}$. WO2 91.0018 
Zuständigkeit des Europäischen Gerichtshofes zu begründen. In den Niederlanden wird deshalb vorgeschlagen, die Vertragsstaaten sollten vollen Gebrauch von den Vorbehalten dahingehend machen, daß eine Verweisung an Vertragsstaaten, welche keine effektiven Verfahrensgarantien entwickelt haben, nicht vorgenommen werden solle $e^{86}$.

Eine derartig differenzierende Lösung scheint aus verfassungsrechtlichen Gründen für die Bundesrepublik nicht möglich zu sein. Vielmehr kann die Bundesregierung erst dann auf die Vorbehaltsregelungen verzichten, wenn in allen Vertragsstaaten einheitliche materielle und insbesondere effektive prozessuale Standards angewandt werden. Meßlatte für eine derartige verfassungspolitische Klärung der Asylfrage könnten die analysierten internationalen Standards sein. Zunächst ist festzuhalten, daß das Bundesverwaltungsgericht zum Kerngehalt des Asylrechts das Verbot der Zurückweisung und Abschicbung in den Verfolgerstaat rechnet. Im übrigen sei Art. 16 Abs. 2 Satz 2 eine offene Norm ${ }^{87}$. Mit Blick auf den Begriff der Verfolgungssicherheit im Drittstaat hat das Gericht überdies wiederholt auf die Schutzbedürftigkeit des Asylsuchenden abgestellt ${ }^{\$ 8}$. Das Erfordernis der Schutzlosigkeit wird also als zusätzliches ungeschriebenes Tatbestandsmerkmal ${ }^{39}$ in die Verfassung hineininterpretiert. Damit sind die dogmatische Strukturen für eine Klärung der Verfassungsfrage deutlich geworden. Zu Recht hat die Bundesregierung wegen der verfassungsrechtlichen Risiken mit dem Gesetzentwurf zur Ratifizierung des Schengener Vertrages den nationalen Vorbehalt geltend gemacht ${ }^{\circ \circ}$. Denn die Klärung einer derart zentralen Verfassungsfrage darf im demokratischen Rechtsstaat nicht der Rechtsprechung, sondern muß dem Verfassungsgeber überlassen bleiben.

Die vertragliche Einbindung der Bundesrepublik setzt einen in etwa vergleichbaren politischen und rechtlichen Kontext voraus. Die konkrete Ausgestaltung effektiver Verfahrensgarantien stößt bereits in EG-Kontext auf kaum lösbare Probleme und dürfte mit Blick auf Staaten wie Polen und crst recht Marokko unlösbar bleiben. Wenn jedoch in allen EG-Staaten einheitliche materielle und prozessuale Mindeststandards eingerichtet worden sind, ist aus verfassungsrechtlicher Sicht die Verweisung eines Asylsuchenden an den zuständigen Vertragsstaat unbedenklich. Hierdurch würde die Schutzwirkung der Asylgarantie in keiner Weise geschwächt. Insbesondere die verfassungsrechtlich verstärkte Pflicht zur Verfahrensgestaltung ${ }^{9 t}$ sowie sämtliche weiteren Garantien blieben für den Bereich der Bundesrepublik wirksam. Damit andererseits in allen Staaten der EG wirksame Verfahrensgarantien eingerichtet werden, ist die verfassungsrechtliche Klarstellung geboten. Die starre Fixierung allein auf die nationale Schutzgarantie hilft keinem Flüchtling außerhalb der Bundesrepublik. Es ist jetzt an der Zeit, sich in Europa einzumischen. Die Umsetzung des Prinzips der einen Chancc setze nämlich eine einheitliche effektive Verfahrensgestaltung in den Staaten der EG voraus. Ohne öffentlichen Druck wird sich insoweit aber nichts bewegen. Bevor aber dicses Ziel nicht erreicht ist, darf die Bundesrepublik ihre völkerrechtliche Verantwortung nicht auf einen anderen Staat übertragen.

86 Roel Fernhour, The legal structure of IGC-treaties and conventions concerning immigrants and refugees, Nijmegen 1992, 5.8 (unveroffentlicht)

87 BVerwGE 49, 202 (205): $69,323(325)$.

88 BVerwGE 69, $289(294) ; 79,347 ; 81,164$.

89 BVerwG, NWwZ $1992,380(381)$.

90 BR, Dr. $121 / 92$, S. 8; BT-Drs. 12/2453; s. auch Debatte des Br, in: 12 WP, S. 72296 ff.

91 BVerfGE $56,216$. 
I. Die Umsetzung des vertraglich vorgegebenen Grundsatzes der einen Chance setzt unabdingbar eine materielle und prozessuale Angleichung der nationalen Asylverfahren voraus.

2. Unabdingbar ist das vertraglich geregelte subjektive Recht auf Zugang zum Verfahren in wenigstens einem Vertragsstaat.

3. Zulässigkeitsverfahren müssen als effektive Schutzgarantien zumindest das verfahrensabhängige Verbleiberecht und das unabhängige Überprüfungsrecht gewährleisten.

4. Mit Hinweis auf den Begriff des Erstaufnahmelandes darf nur dann der Zugang zum Verfahren versperrt werden, wenn der Asylsuchende in einem Drittstaat konkrete Schutzalternativen gehabt hat und hinreichend gesichert ist, daß er dort dauerhaften Schutz oder zumindest Anspruch auf Prüfung seines Asylbegehrens erhält.

5. Beförderungsverbote verletzen die zwingende völkerrechtliche Regel des Refoulement-Verbotes. Bei der Gestaltung der curopäischen Asylpolitik ist daher auf dieses Mittel der Fluchtverhinderung zu verzichten.

6. Als unabdingbare prozessuale Mindestgarantien müssen nationale Asylverfahren klare Anweisungen an die Grenz- und Ausländerbehörden mit Blick auf das Refoulement-Verbot enthalten, muß eine qualifizierte zentrale Asylbehörde jeden Asylantrag durch persönliche Anhörung prüfen und ist eine Überprüfung mit verfahrensabhängigem Verbleiberecht - regelmäßig in einem gerichtsförmigen Verfahren oder zumindest durch eine unabhängige und unvoreingenommene Beschwerdeinstanzsicherzustellen.

6. Erst wenn eine derartige prozessuale und materielle Harmonisierung in allen EGStaaten erreicht ist, ist aus verfassungsrechtlicher Sicht gegen die Verweisung cines Asylsuchenden an den zuständigen Staat nichts zu erinnern. 